\title{
VARIATIONAL ANALYSIS FOR SIMULATING FREE-SURFACE FLOWS IN A POROUS MEDIUM
}

\section{SHABBIR AHMED AND CHARLES COLLINS}

Received 29 January 2003 and in revised form 25 March 2003

A variational formulation has been developed to solve a parabolic partial differential equation describing free-surface flows in a porous medium. The variational finite element method is used to obtain a discrete form of equations for a two-dimensional domain. The matrix characteristics and the stability criteria have been investigated to develop a stable numerical algorithm for solving the governing equation. A computer programme has been written to solve a symmetric positive definite system obtained from the variational finite element analysis. The system of equations is solved using the conjugate gradient method. The solution generates time-varying hydraulic heads in the subsurface. The interfacing free surface between the unsaturated and saturated zones in the variably saturated domain is located, based on the computed hydraulic heads. Example problems are investigated. The finite element solutions are compared with the exact solutions for the example problems. The numerical characteristics of the finite element solution method are also investigated using the example problems.

\section{Introduction}

The parabolic partial differential equations are solved to describe the variations of hydraulic heads in groundwater systems including unsaturated (above groundwater table) and saturated (below groundwater table) zones. Galerkin finite element methods were widely used by researchers for solving unsaturated and/or saturated flow and contaminant transport problems $[6,9,11,12,13,16]$. In these works, the Galerkin method was used directly by employing appropriate shape 
functions. The mathematical and numerical analyses associated with the continuity, stability, and growth of solution was not investigated in greater detail in these works. For a better understanding of the application of the finite element method for solving a parabolic equation in the groundwater system, it is important to investigate the mathematical and numerical aspects of the techniques for ensuring smooth and stable solutions.

In earlier studies, Galerkin approximations with error and continuity analyses were performed for general second-order parabolic partial differential equations $[2,10]$. The norm error estimates for semidiscrete Galerkin finite element methods for parabolic problems were analyzed as well [14]. In that study, a fully discrete approximation of the solutions of diffusion equations was presented to investigate the density or concentration of fluids in fissured media. Convergence of the method was proved in addition to the numerical experiments to confirm theoretical results. In another study, an automatic adaptation of finite element approximation space with time for the solution of a general class of parabolic linear equations was described [7]. In all these analyses, mathematical and numerical investigations were done to establish the finite element formulations for the class of parabolic partial differential equations. But, the attempts were not made to implement the formulation for solving the real-world physical problems described by the parabolic partial differential equations.

In the present paper, mathematical and numerical analyses are performed to solve a parabolic partial differential equation representing variably saturated flow in a porous medium. Variational problem formulation is investigated for generating finite element solutions. The growth estimates and matrix characteristics for the fully discrete method based on backward Euler formulation are investigated. The backward Euler formulation is implemented in a computer programme to simulate the time history of hydraulic heads for variably saturated flow in a porous medium. The time history of the free surface representing groundwater table in a porous medium is also located, based on the simulated hydraulic heads.

\section{Variational problem formulation}

The governing equation describing variably saturated flow in the subsurface can be written with the initial and boundary conditions (see Figure 2.1) as follows [1, 3]:

$$
\begin{gathered}
E(\psi) \frac{\partial \varphi}{\partial t}=\nabla \cdot[K(\psi) \nabla \varphi]+q \quad \text { in } \Omega \times I, \\
\varphi=\varphi_{d} \quad \text { on } \Gamma_{d} \times I,
\end{gathered}
$$




$$
\begin{gathered}
K(\psi) \frac{\partial \varphi}{\partial n}=g \quad \text { on } \Gamma_{n} \times I, \\
\varphi(x, 0)=\varphi^{0} \quad \text { in } \Omega .
\end{gathered}
$$

Here boundary $\Gamma$ is divided such that $\Gamma=\Gamma_{n} \cup \Gamma_{d}\left(\Gamma_{n}=\right.$ Neumann boundary, $\Gamma_{d}=$ Dirichlet boundary). The domain $\Omega$ is a two-dimensional bounded region. The time interval $I=(0, T)$, where $T$ is a given time. The dependent variable $\varphi$ represents hydraulic head in the subsurface which is the sum of pressure head $(\psi)$ and elevation $z$. The expression $\varphi(x, 0)=\varphi^{0}$ gives the distribution of initial hydraulic heads in the domain.

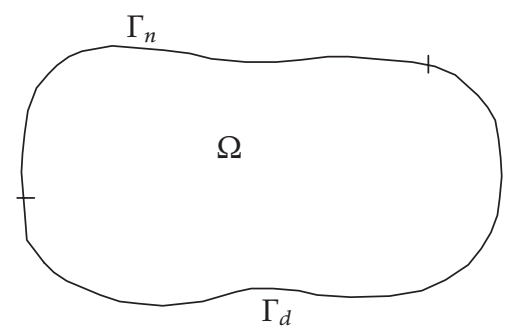

FIGURE 2.1. A domain with boundary conditions.

The nonlinear functions $E(\psi)$ and $K(\psi)$ represent soil-moisture capacity and hydraulic conductivity in the subsurface. These are evaluated assuming that the value of $\psi$ is known. The actual value of $\psi$ will be described later. The functions $E(\psi)$ and $K(\psi)$ are used, based on the empirical formulations as a function of pressure head $\psi[1,15]$.

For the aforementioned initial boundary value problem (IBVP), some function spaces are defined to obtain a variational formulation for problem as described in (2.1). We define function spaces on the domain $\Omega \subset$ $\mathbb{R}^{2}$, where $\mathbb{R}^{2}$ defines a two-dimensional plane, as follows:

$$
\begin{aligned}
L_{2}(\Omega) & =\left\{v: v \text { is defined on } \Omega \text { and } \int_{\Omega} v^{2} d x<\infty\right\}, \\
H^{1}(\Omega) & =\left\{v \in L_{2}(\Omega): \nabla v \in L_{2}(\Omega)\right\}, \\
V & =\left\{v \in H^{1}(\Omega): v=\varphi_{d} \text { on } \Gamma_{d}\right\}, \\
V^{0} & =\left\{v \in H^{1}(\Omega): v=0 \text { on } \Gamma_{d}\right\} .
\end{aligned}
$$

Here $L_{2}(\Omega), H^{1}(\Omega)$, and $V^{0}$ are Hilbert spaces with the appropriate scalar products. 
380 Variational analysis for simulating free-surface flows

Let $\varphi_{d}$ be extended to all of $\Omega$. Set $\varphi=\varphi_{d}+u$ in (2.1) to obtain homogeneity in the Dirichlet boundary conditions as follows:

$$
\begin{gathered}
E(\psi) \frac{\partial u}{\partial t}=\nabla \cdot[K(\psi) \nabla u]+\tilde{q} \quad \text { in } \Omega \times I, \\
u=0 \quad \text { on } \Gamma_{d} \times I, \\
K(\psi) \frac{\partial u}{\partial n}=g \quad \text { on } \Gamma_{n} \times I, \\
u(x, 0)=u^{0} \quad \text { in } \Omega,
\end{gathered}
$$

where

$$
\tilde{q}=q+\nabla \cdot\left(K(\psi) \nabla \varphi_{d}\right)-E(\psi) \frac{\partial \varphi_{d}}{\partial t}
$$

Here $u(t) \in V^{0}$.

Without loss of generality, we can take $\varphi=0$ on $\Gamma_{d}$ and use problem (2.1) with $\varphi_{d}=0$. Multiplying (2.1) by a test function $v \in V^{0}$, integrating over $\Omega$, and using Green's formula, we obtain

$$
\int_{\Omega} E \frac{\partial \varphi}{\partial t} v d \Omega=\int_{\Gamma} K \frac{\partial \varphi}{\partial n} v d \Gamma-\int_{\Omega} K \nabla \varphi \cdot \nabla v d \Omega+\int_{\Omega} q v d \Omega
$$

Therefore, the variational formulation can be defined as follows:

$(\mathrm{V})$ to find $\varphi(t) \in V^{0}$ such that

$$
\begin{gathered}
(E \dot{\varphi}(t), v)+a(\psi ; \varphi(t), v)=(q(t), v)+\langle g(t), v\rangle_{\Gamma_{n}} \quad \forall v \in V^{0}, t \in I \\
(\varphi(\cdot, 0), v)=\left(\varphi^{0}, v\right) \quad \forall v \in V^{0}
\end{gathered}
$$

where

$$
\begin{aligned}
a(\psi ; v, w) & =\int_{\Omega} K(\psi) \nabla v \cdot \nabla w d \Omega, \\
(E \dot{\varphi}(t), v) & =\int_{\Omega} E \dot{\varphi}(t) v d \Omega, \\
(q(t), v) & =\int_{\Omega} q(t) v d \Omega, \\
\langle g(t), v\rangle_{\Gamma_{n}} & =\int_{\Gamma_{n}} g(t) v d \Gamma .
\end{aligned}
$$


Equations (2.6) give a variational formulation of the original problem. Therefore, $\varphi$ is a solution of problem (2.1) given by the variational problem as shown in (2.6). The variational formulation (2.6) can be also rewritten as

$$
a(\psi ; \varphi, v)=(h(t), v)+\langle g, v\rangle
$$

where

$$
(h(t), v)=(q(t)-E \dot{\varphi}(t), v) .
$$

\section{Discretization in space and time}

We consider a triangulation of the domain $\Omega$, that is, we subdivide $\Omega$ into a set $T_{h}$ of nonoverlapping triangles $K_{i}(i=1,2, \ldots, m)$ such that

$$
\Omega=\bigcup_{K \in T_{h}} K=K_{1} \cup K_{2} \cup \cdots \cup K_{m} .
$$

Let $V_{h}^{0}$ be a finite-dimensional subspace of the space $V^{0}$ consisting of piecewise linear functions. We define $P_{1}(K)$ as the space of linear functions on $K$, that is,

$$
P_{1}(K)=\{v: v \text { is a polynomial of degree } \leq 1 \text { on } K\} .
$$

Here

$$
V_{h}^{0}=\left\{v \in V^{0}:\left.v\right|_{k} \in P_{1}(k) \text { and } v \text { is continuous at the nodes, } \forall K \in T_{h}\right\}
$$

Let the basis functions for $P_{1}(K)$ of the finite-dimensional subspace $V_{h}^{0}$ of $V^{0}$ be $\left\{L_{1}, L_{2}, L_{3}, \ldots, L_{M}\right\}$.

For $\Omega \subset \mathbb{R}^{2}$ and $V_{h}^{0}$ as described, the fully discrete analogue of the variational problem (2.6) can be obtained using backward Euler method [8]. Let $0=t_{0}<t_{1}<t_{2}<\cdots<t_{N}=T$ denote a partitioning of $I$ so that $I_{n}=\left(t_{n-1}, t_{n}\right)$. Let $k_{n}=t_{n}-t_{n-1}$ be the time step size. Let the values of $E(\psi)$ and $K(\psi)$ be evaluated based on the values of $\psi$ at previous time step $n-1$. Using the backward Euler method, the derivative $\dot{\varphi}\left(t_{n}\right)$ in (2.6) can be replaced by the difference quotient $\left(\varphi_{h}^{n}-\varphi_{h}^{n-1}\right) / k_{n}$ with the discretization error $O\left(k_{n}\right)$. Therefore, the fully discrete variational problem 
382 Variational analysis for simulating free-surface flows

(2.6) can be written as follows: to find $\varphi_{h}^{n} \in V_{h}^{0}, n=1,2, \ldots, N$, such that

$$
\begin{aligned}
\left(E\left(\varphi_{h}^{n}-\varphi_{h}^{n-1}\right), v\right)+k_{n} a\left(\psi ; \varphi_{h}^{n}, v\right) & =k_{n}\left(q\left(t_{n}\right), v\right)+k_{n}\left\langle g\left(t_{n}\right), v\right\rangle \quad \forall v \in V_{h}^{0}, \\
\left(\varphi_{h}(\cdot, 0), v\right) & =\left(\varphi^{0}, v\right) \quad \forall v \in V_{h}^{0} .
\end{aligned}
$$

Writing $\varphi_{h}(x, t)$ as $\varphi_{h}(x, t)=\sum_{i=1}^{M} \xi_{i}(t) L_{i}(x), \xi_{i}(t) \in \mathbb{R}$, and taking $v=L_{j}$, we can write (3.4) in a matrix form as

$$
\left(B^{n-1}+k_{n} A^{n-1}\right) \xi^{n}=B^{n-1} \xi^{n-1} \pm k_{n} F\left(t_{n}\right),
$$

where

$$
\begin{aligned}
& B=\left(b_{i j}\right), \quad b_{i j}=\int_{\Omega} E\left(\psi^{n-1}\right) L_{i} L_{j} d \Omega, \\
& A=\left(a_{i j}\right), \quad a_{i j}=\int_{\Omega} K\left(\psi^{n-1}\right) \nabla L_{i} \cdot \nabla L_{j} d \Omega, \\
& F=\left(f_{i}\right), \quad f_{i}=\int_{\Omega} q L_{i} d \Omega+\int_{\Gamma_{n}} g L_{i} d \Gamma .
\end{aligned}
$$

\section{Matrix characteristics}

It is necessary to prove certain conditions associated with the variational problem [8]. The bilinear form $a(\cdot, \cdot)$ defines the stiffness matrix $A$. The conditions associated with the bilinear form and the right-hand side of (2.8) are the following:

(i) $a(\cdot, \cdot)$ is symmetric;

(ii) $a(\cdot, \cdot)$ is continuous, that is, there exists $\gamma>0$ such that $|a(v, w)| \leq$ $\gamma\|v\|_{v}\|w\|_{v}$ for all $v, w \in V^{0}$

(iii) $a(\cdot, \cdot)$ is $V$-elliptic, that is, there exists $\alpha>0$ such that $a(v, v) \geq$ $\alpha\|v\|_{v}^{2}$ for all $v \in V^{0}$

(iv) the right-hand side of (2.8) should be continuous. Let $\ell(v)=$ $(h, v)+\langle g, v\rangle$ and $\ell(v)$ is continuous, that is, there exists $\Lambda>0$ such that $|L(v)| \leq \Lambda\|v\|_{v}$ for all $v \in V^{0}$.

Proof of (i). Here

$$
a(\psi ; v, w)=\int_{\Omega} K(\psi) \nabla v \cdot \nabla w d \Omega
$$

Now

$$
a(\psi ; w, v)=\int_{\Omega} K(\psi) \nabla w \cdot \nabla v d \Omega
$$


Since $K \nabla v \cdot \nabla w=K \nabla w \cdot \nabla v, a(\psi ; v, w)=a(\psi ; w, v)$. This implies that $a(\cdot ; \cdot, \cdot)$ is symmetric.

Proof of (ii). We introduce the scalar products and norm in $V^{0}$ :

$$
\begin{aligned}
(v, w) & =\int_{\Omega} K(\psi) \nabla v \cdot \nabla w d \Omega \\
\|v\| & =\left(\int_{\Omega} K(\psi) \nabla v \cdot \nabla v d \Omega\right)^{1 / 2}
\end{aligned}
$$

Here

$$
a(\psi ; v, w)=\int_{\Omega} K(\psi) \nabla v \cdot \nabla w d \Omega .
$$

Now

$$
a(\psi ; v, v)=\int_{\Omega} K(\psi) \nabla v \cdot \nabla v d \Omega=\|v\|^{2}
$$

By Cauchy's inequality,

$$
a(\psi ; v, w) \leq a(\psi ; v, v)^{1 / 2} a(\psi ; w, w)^{1 / 2}
$$

which can be written as

$$
a(\psi ; v, w) \leq\|v\|\|w\|
$$

Therefore, $a(\psi ; v, w)$ is continuous.

Proof of (iii). As before,

$$
a(\psi ; v, v)=\int_{\Omega} K(\psi) \nabla v \cdot \nabla v d \Omega=\|v\|^{2}
$$

This implies that $a(\psi ; v, v)$ is $V$-elliptic.

Proof of (iv). Here, $\ell(v)=(h, v)+\langle g, v\rangle$.

By Cauchy's inequality and the Poincaré inequality, there is a constant C such that

$$
\begin{aligned}
& |(h, v)| \leq C\|h\|_{L_{2}(\Omega)}\|v\|, \\
& |\langle g, v\rangle| \leq C\|g\|_{L_{2}(\Gamma)}\|v\| .
\end{aligned}
$$


384 Variational analysis for simulating free-surface flows

Therefore,

$$
|\ell(v)| \leq C\left(\|h\|_{L_{2}(\Omega)}+\|g\|_{L_{2}(\Gamma)}\right)\|v\| \quad \text { or } \quad|\ell(v)| \leq \Lambda\|v\| .
$$

Therefore, $\ell(v)$ is continuous with $\Lambda=C\left(\|h\|_{L_{2}(\Omega)}+\|g\|_{L_{2}(\Gamma)}\right)$.

Analysis of matrix $B$

Here

$$
b(\psi ; v, w)=\int_{\Omega} E(\psi) v w d \Omega .
$$

Now

$$
b(\psi ; w, v)=\int_{\Omega} E(\psi) w v d \Omega .
$$

Since $v w=w v, b(\psi ; v, w)=b(\psi ; w, v)$. This implies that $b(\cdot \because, \cdot)$ is symmetric. It can be written as

$$
b(\psi ; v, v)=b\left(\psi ; \sum_{i=1}^{m} \eta_{i} \varphi_{i}, \sum_{j=1}^{m} \eta_{j} \varphi_{j}\right)=\sum_{i, j=1}^{m} \eta_{i} b\left(\psi ; \varphi_{i}, \varphi_{j}\right) \eta_{j}=\eta \cdot B \eta .
$$

Also,

$$
b(\psi ; v, v)=\int_{\Omega} E(\psi) v^{2} d \Omega .
$$

From Poincaré's inequality, there exists a constant $C>0$ such that

$$
\int_{\Omega} v^{2} d \Omega \leq C \int_{\Omega}|\nabla v|^{2} d \Omega \quad \forall v \in V^{0}
$$

Since $E(\psi)>0$,

$$
\int_{\Omega} E(\psi) v^{2} d \Omega \leq C \int_{\Omega} E(\psi)|\nabla v|^{2} d \Omega>0 \quad \forall v \in V^{0} .
$$

From (4.14) and (4.16), we get that

$$
\eta \cdot B \eta=b(\psi ; v, v)>0 \Longrightarrow B \text { is positive definite. }
$$


The matrix $B$ was proved to be symmetric earlier, so $B$ is a symmetric positive definite matrix. By the proofs of (i), (ii), and (iii), matrix $A$ is symmetric positive definite. Therefore, $B+k_{n} A$ is a symmetric positive definite matrix. This implies that all the eigenvalues of $B+k_{n} A$ must be real and positive. For a symmetric positive definite system, conjugate gradient method is used. In this method, an efficient algorithm is used by operating on the half of the bandwidth of the symmetric positive definite coefficient matrix.

\section{Growth estimate}

With $q=0$ and $g=0$, a growth estimate is done to prove the reliability of the finite element formulation. It can be proved that

$$
\max _{n}\left\|\varphi_{h}^{n}-\varphi_{h}^{n-1}\right\| \leq \frac{C\left\|\varphi^{0}\right\|}{\sqrt{E}}\left(1+\log \frac{T}{k_{1}}\right)^{1 / 2} .
$$

In (3.4), with $q=0$ and $g=0$, we can write

$$
\left(E\left(\varphi_{h}^{n}-\varphi_{h}^{n-1}\right), v\right)+k_{n} a\left(\psi ; \varphi_{h^{\prime}}^{n} v\right)=0 .
$$

Setting $v=t_{n}\left(\varphi_{h}^{n}-\varphi_{h}^{n-1}\right)$, summing over $n$, and rearranging, we get

$$
\sum_{n=1}^{N} E t_{n}\left\|\frac{\varphi_{h}^{n}-\varphi_{h}^{n-1}}{k_{n}}\right\|^{2} k_{n}=\sum_{n=1}^{N} a\left(\psi ; \varphi_{h}^{n}-\varphi_{h}^{n-1}, k_{n} \varphi_{h}^{n-1}\right) .
$$

Since $a(\because \cdot, \cdot)$ is continuous, for a constant $\gamma>0$,

$$
a\left(\psi ; \varphi_{h}^{n}-\varphi_{h}^{n-1}, k_{n} \varphi_{h}^{n-1}\right) \leq \gamma\left\|\varphi_{h}^{n}-\varphi_{h}^{n-1}\right\|\left\|k_{n} \varphi_{h}^{n-1}\right\| .
$$

Since $\left\|\varphi_{h}^{n}-\varphi_{h}^{n-1}\right\| \leq\left\|\varphi^{0}\right\|$ and $\left\|\varphi_{h}^{n-1}\right\| \leq\left\|\varphi^{0}\right\|$,

$$
a\left(\psi ; \varphi_{h}^{n}-\varphi_{h}^{n-1}, k_{n} \varphi_{h}^{n-1}\right) \leq \gamma k_{n}\left\|\varphi^{0}\right\|^{2} .
$$

Substituting (5.5) in (5.3), we get

$$
\left(\sum_{n=1}^{N} E t_{n}\left\|\frac{\varphi_{h}^{n}-\varphi_{h}^{n-1}}{k_{n}}\right\|^{2} k_{n}\right)^{1 / 2} \leq C\left\|\varphi^{0}\right\|, \quad C=\sqrt{\gamma k_{n}} .
$$


386 Variational analysis for simulating free-surface flows

Now

$$
\sum_{n=1}^{N}\left\|\frac{\varphi_{h}^{n}-\varphi_{h}^{n-1}}{k_{n}}\right\| k_{n}
$$

can be written as

$$
\sum_{n=1}^{N}\left\|\frac{\varphi_{h}^{n}-\varphi_{h}^{n-1}}{k_{n}}\right\| k_{n}=\sum_{n=1}^{N}\left\{\sqrt{E}\left\|\frac{\varphi_{h}^{n}-\varphi_{h}^{n-1}}{k_{n}}\right\| \sqrt{k_{n} t_{n}}\right\} \sqrt{\frac{k_{n}}{E t_{n}}} .
$$

Then, by Cauchy's inequality,

$$
\sum_{n=1}^{N}\left\|\frac{\varphi_{h}^{n}-\varphi_{h}^{n-1}}{k_{n}}\right\| k_{n} \leq\left(\sum_{n=1}^{N} E t_{n}\left\|\frac{\varphi_{h}^{n}-\varphi_{h}^{n-1}}{k_{n}}\right\|^{2} k_{n}\right)^{1 / 2}\left(\frac{1}{\sqrt{E}}\right)\left(\sum_{n=1}^{N} \frac{k_{n}}{t_{n}}\right)^{1 / 2}
$$

From (5.6) and (5.9), we get

$$
\sum_{n=1}^{N}\left\|\frac{\varphi_{h}^{n}-\varphi_{h}^{n-1}}{k_{n}}\right\| k_{n} \leq \frac{C\left\|\varphi^{0}\right\|}{\sqrt{E}}\left(\sum_{n=1}^{N} \frac{k_{n}}{t_{n}}\right)^{1 / 2}
$$

The last summation term in (5.10) can be expressed as

$$
\sum_{n=1}^{N} \frac{k_{n}}{t_{n}} \approx \int_{0}^{T} \frac{1}{t} d t=\int_{0}^{t_{1}} \frac{1}{t} d t+\int_{t_{1}=k_{1}}^{T} \frac{1}{t} d t
$$

By approximating $\int_{0}^{t_{1}}(1 / t) d t \approx 1$, we can write

$$
\sum_{n=1}^{N} \frac{k_{n}}{t_{n}} \leq 1+\log \frac{T}{k_{1}}
$$

Therefore, (5.10) can be written as

$$
\sum_{n=1}^{N}\left\|\frac{\varphi_{h}^{n}-\varphi_{h}^{n-1}}{k_{n}}\right\| k_{n} \leq \frac{C\left\|\varphi^{0}\right\|}{\sqrt{E}}\left(1+\log \frac{T}{k_{1}}\right)^{1 / 2}
$$


from which we get that

$$
\max _{n}\left\|\varphi_{h}^{n}-\varphi_{h}^{n-1}\right\| \leq \frac{C\left\|\varphi^{0}\right\|}{\sqrt{E}}\left(1+\log \frac{T}{k_{1}}\right)^{1 / 2} .
$$

Similarly, following a stability estimate, the time step control can be adopted as follows [4].

Suppose that $\delta>0$ is a given tolerance and suppose that we want the discrete solution $\varphi_{h}(t)$ to satisfy

$$
\max _{t \leq t_{N}}\left\|\varphi(t)-\varphi_{h}(t)\right\| \leq \delta .
$$

The time step $k_{n}$ can be chosen so that, for $n=1,2, \ldots, N$,

$$
k_{n} \max _{t \in I}\|\dot{\varphi}\| \approx \frac{\delta}{C} .
$$

It is assumed that the size of the constant is known approximately (up to, say, a factor 2). The above condition can be replaced by the condition

$$
\left\|\varphi_{h}^{n}-\varphi_{h}^{n-1}\right\| \approx \frac{\delta}{C}
$$

Based on the above conditions, an algorithm can be adopted for choosing $k_{n}$, assuming that $\varphi_{h}^{n-1}$ has been computed.

\section{Examples to evaluate numerical characteristics}

Two numerical examples are described to demonstrate the application of the variational finite element analysis to simulate the hydraulic heads and free surface in a porous medium. The analytical solutions are obtained for the two-dimensional example problems using some simplifying assumptions. The following assumptions are considered:

(i) the domain is assumed to be rectangular in shape;

(ii) the medium is assumed to be homogeneous and isotropic;

(iii) the hydraulic conductivity is assumed to be constant with an effective value for the medium.

Based on the above assumptions, the governing equation can be written as

$$
\frac{\partial \varphi}{\partial t}=\alpha \Delta \varphi+q \quad \text { for } \varphi \in C^{2}(\Omega) \cup C^{1}(\Gamma) .
$$

Here $\alpha=K / E$. Two analytical solutions are obtained for different 
388 Variational analysis for simulating free-surface flows

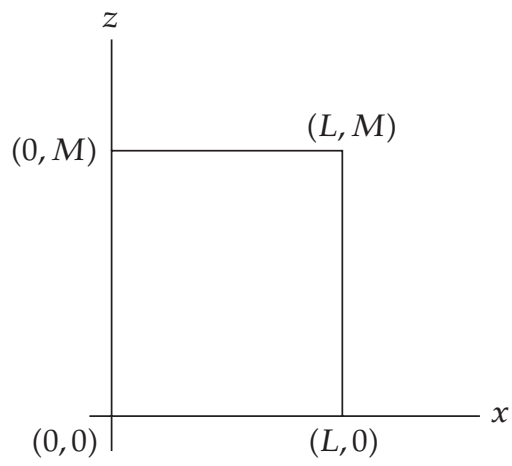

FIGURE 6.1. Computational domain for the example problems.

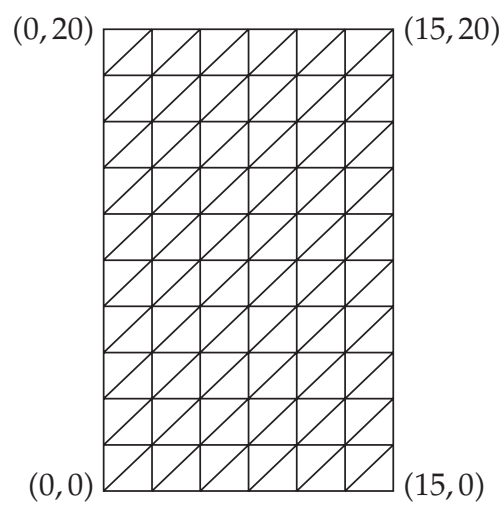

FIGURE 6.2. Finite elements in the computational domain.

boundary conditions in a rectangular domain as shown in Figure 6.1. The method of separation of variables is used to derive the analytical solution for both problems. For the finite element solutions, the domain is discretized using triangles as shown in Figure 6.2. The hydraulic conductivity is assumed to be $1 \mathrm{ft} / \mathrm{d}$ and the storage term is assumed to be 0.025 .

\subsection{Example 1}

In the first example, Dirichlet boundary conditions are used on all sides of the rectangular domain (Figure 6.1). The boundary conditions are

$$
\begin{array}{rlrl}
\varphi(0, z, t) & =f_{1}(z), & & 0<z<M, t>0, \\
\varphi(x, 0, t) & =f_{2}(x), & & 0<x<L, t>0, \\
\varphi(L, z, t) & =f_{3}(z), & 0<z<M, t>0, \\
\varphi(x, M, t) & =f_{4}(x), & & 0<x<L, t>0 .
\end{array}
$$


Here, $f_{1}(z), f_{2}(x), f_{3}(z)$, and $f_{4}(x)$ can be expressed as follows:

$$
\begin{aligned}
& f_{1}(z)=a+b \frac{z}{M^{\prime}} \\
& f_{2}(x)=a+\frac{b x}{L}\left(1-\frac{x}{L}\right), \\
& f_{3}(z)=a+c \frac{z}{M^{\prime}} \\
& f_{4}(x)=a-\frac{b x}{L}\left(1-\frac{x}{L}\right),
\end{aligned}
$$

where $a, b, c, d \in \mathbb{R}$.

Using the method of separation of variables, the solution for the hydraulic head $\varphi(x, z, t)$ for the Dirichlet problem is written as

$$
\varphi(x, z, t)=\sum_{n=1}^{\infty}\left[\sum_{m=1}^{\infty} K_{m n}(t) \sin \frac{m \pi z}{M}+w(z)\right] \sin \frac{n \pi x}{L}+\zeta(x, z),
$$

where

$$
\begin{aligned}
K_{m n}(t) & =\int_{0}^{t} e^{-\alpha \lambda_{m n}(t-\tau)} q_{m n}(z, \tau) d \tau+g e^{-\alpha \lambda_{m n} t}, \\
q_{m n} & =\frac{2}{M} \int_{0}^{M} \hat{q} \sin \frac{m \pi z}{M} d z, \quad m=1,2,3, \ldots, \\
\hat{q} & =\tilde{q}-\alpha \lambda_{n} w(z), \quad n=1,2,3, \ldots, \\
\tilde{q} & =\frac{2}{L} \int_{0}^{L} q \sin \frac{n \pi x}{L} d x, \quad n=1,2,3, \ldots, \\
\lambda_{n} & =\left(\frac{n \pi}{L}\right)^{2}, \quad n=1,2,3, \ldots, \\
\lambda_{m n} & =\lambda_{m}+\lambda_{n}, \quad m=1,2,3, \ldots, n=1,2,3, \ldots, \\
\lambda_{m} & =\left(\frac{m \pi}{M}\right)^{2}, \quad m=1,2,3, \ldots, \\
g & =\frac{2}{M} \int_{0}^{M} u^{0} \sin \frac{m \pi z}{M} d z, \quad m=1,2,3, \ldots, \\
u^{0} & =v^{0}-w(z), \quad v^{0}=\frac{2}{L} \int_{0}^{L}\left(\varphi^{0}-\zeta(x, z)\right) \sin \frac{n \pi x}{L} d x,
\end{aligned}
$$


390 Variational analysis for simulating free-surface flows

$$
\begin{aligned}
\zeta(x, z) & =a+b \frac{z}{M}\left(1-\frac{x}{L}\right)+c \frac{x z}{L M}, \\
w(z) & =\left(1-\frac{z}{M}\right) v(0, t)+\frac{z}{M} v(M, t), \\
v(0, t) & =\frac{2}{L} \int_{0}^{L}\left(f_{2}(x)-a\right) \sin \frac{n \pi x}{L} d x, \\
v(M, t) & =\frac{2}{L} \int_{0}^{L}\left(f_{4}(x)-a-b\left(1-\frac{x}{L}\right)-c \frac{x}{L}\right) \sin \frac{n \pi x}{L} d x .
\end{aligned}
$$

The results obtained for both the numerical and analytical solutions at $(x, z)=(7.5,10)$ in the domain for the Dirichlet problem are shown in Figure 6.3. It is observed that the results obtained from the variational finite element solutions match well with the analytical solutions for the Dirichlet boundary conditions.

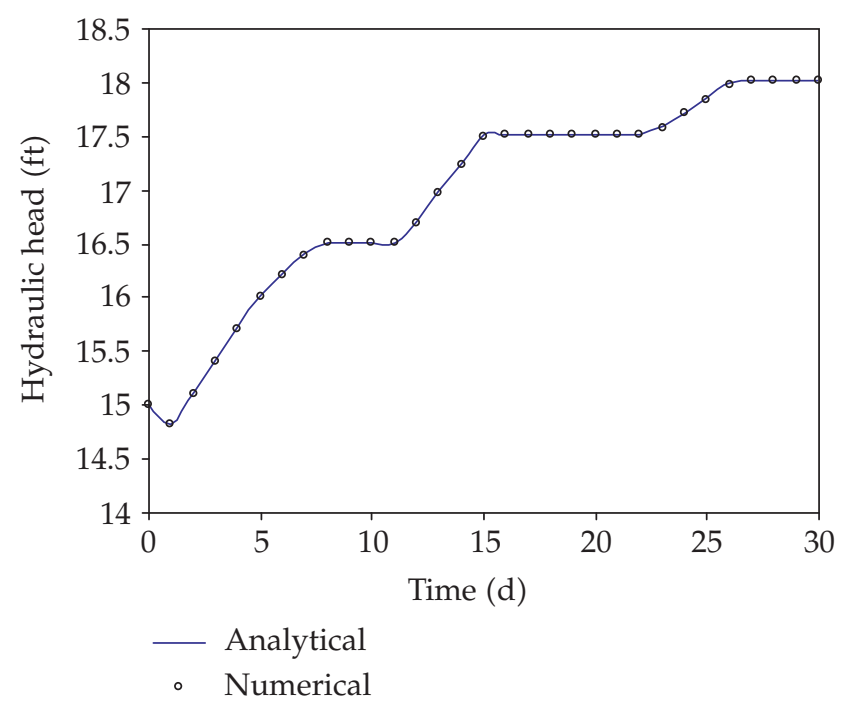

FIGURE 6.3. Finite element and analytical solutions for the Dirichlet problem.

\subsection{Example 2}

In the mixed problem, both Dirichlet and Neumann boundary conditions are applied on the sides of the two-dimensional rectangular domain (Figure 6.1). The boundary conditions used in the mixed problem 
are as follows:

$$
\begin{array}{rlrl}
\varphi(0, z, t) & =f_{1}(z), & & 0<z<M, t>0, \\
\varphi(x, 0, t) & =f_{2}(x), & 0<x<L, t>0, \\
\varphi_{x}(L, z, t) & =f_{3}(z), & 0<z<M, t>0, \\
\varphi_{z}(x, M, t) & =f_{4}(x), & 0<x<L, t>0 .
\end{array}
$$

Here $f_{1}(z), f_{2}(x), f_{3}(z)$, and $f_{4}(x)$ can be expressed as follows:

$$
\begin{aligned}
& f_{1}(z)=a+b \frac{z}{M}, \quad f_{2}(x)=c-\frac{d x}{L}, \\
& f_{3}(z)=\frac{b z}{L M}, \quad f_{4}(x)=b-\frac{d x}{L} .
\end{aligned}
$$

The initial condition is expressed as follows:

$$
\varphi(x, z, 0)=\varphi^{0}, \quad 0<x<L, 0<z<M .
$$

On using the method of separation of variables, the solution for the hydraulic head $\varphi(x, z, t)$ for the mixed problem is written as

$$
\begin{aligned}
\varphi(x, z, t)=\sum_{n=0}^{\infty} & {\left[\sum_{m=0}^{\infty} K_{m n}(t) \sin \frac{(2 m+1) \pi z}{2 M}+w(z)\right] } \\
& \times \sin \frac{(2 n+1) \pi x}{2 L}+\zeta(x, z),
\end{aligned}
$$

where

$$
\begin{aligned}
K_{m n}(t) & =\int_{0}^{t} e^{-\alpha \lambda_{m n}(t-\tau)} q_{m n}(z, \tau) d \tau+g e^{-\alpha \lambda_{m n} t}, \quad m=0,1,2, \ldots, \\
n=0,1,2, \ldots, & \\
q_{m n} & =\frac{2}{M} \int_{0}^{M} \hat{q} \sin \frac{(2 m+1) \pi z}{2 M} d z, \quad m=0,1,2, \ldots, \\
\hat{q} & =\tilde{q}-\alpha \lambda_{n} w(z), \\
\tilde{q} & =\frac{2}{L} \int_{0}^{L}\left[q-\frac{\alpha b z}{L^{2} M}\right] \sin \frac{(2 n+1) \pi x}{2 L} d x, \quad n=0,1,2, \ldots, \\
\lambda_{n} & =\left(\frac{(2 n+1) \pi}{2 L}\right)^{2}, \quad n=0,1,2,3, \ldots,
\end{aligned}
$$


Variational analysis for simulating free-surface flows

$$
\begin{aligned}
\lambda_{m n} & =\lambda_{m}+\lambda_{n}, \quad m=0,1,2, \ldots, n=0,1,2, \ldots, \\
\lambda_{m} & =\left(\frac{(2 m+1) \pi}{2 M}\right)^{2}, \quad m=0,1,2,3, \ldots, \\
g & =\frac{2}{M} \int_{0}^{M} u^{0} \sin \frac{(2 m+1) \pi z}{2 M} d z, \quad m=0,1,2, \ldots, \\
u^{0} & =v^{0}-w(z), \quad v^{0}=\frac{2}{L} \int_{0}^{L}\left(\varphi^{0}-\zeta(x, z)\right) \sin \frac{(2 n+1) \pi x}{2 L} d x, \\
\zeta(x, z) & =a+b \frac{z}{M}\left(1-\frac{x^{2}}{2 L^{2}}\right), \\
w(z) & =v(0, t)+\frac{z^{2}}{2 M} v_{z}(M, t), \\
v(0, t) & =\frac{2}{L} \int_{0}^{L}\left(f_{2}(x)-a\right) \sin \frac{(2 n+1) \pi x}{2 L} d x, \\
v_{z}(M, t) & =\frac{2}{L} \int_{0}^{L}\left[f_{4}(x)-\frac{b}{M}\left(1-\frac{x^{2}}{2 L^{2}}\right)\right] \sin \frac{(2 n+1) \pi x}{2 L} d x
\end{aligned}
$$

The results obtained for both the numerical and analytical solutions at $(x, z)=(7.5,10)$ in the domain for the mixed problem are shown in Figure 6.4. The variational finite element solutions match well with the analytical solutions for the mixed problem.

\subsection{Simulation characteristics}

The relative errors $\epsilon$ for the simulations give a relative measure of the absolute deviation from the exact solution. It is computed as follows:

$$
\epsilon=\left|\frac{\varphi-\varphi_{h}}{\varphi}\right| .
$$

Here $\varphi$ and $\varphi_{h}$ are the analytical and numerical solutions, respectively. The relative errors for the point $(x, z)=(7.5,10)$ are shown in Figure 6.5. The variations of errors in other computational points in the domain are found to be similar. A maximum relative error of less than about 0.5 percent is obtained for the numerical solutions for both the Dirichlet and mixed problems. It is observed that the errors do not amplify or grow in an unbounded fashion with time. The relative error $\epsilon$ varies such that $\epsilon<M$ (here $M \approx 0.005$ ).

As revealed in the analysis of matrix characteristics, the variational formulation for this problem generated a symmetric positive definite 


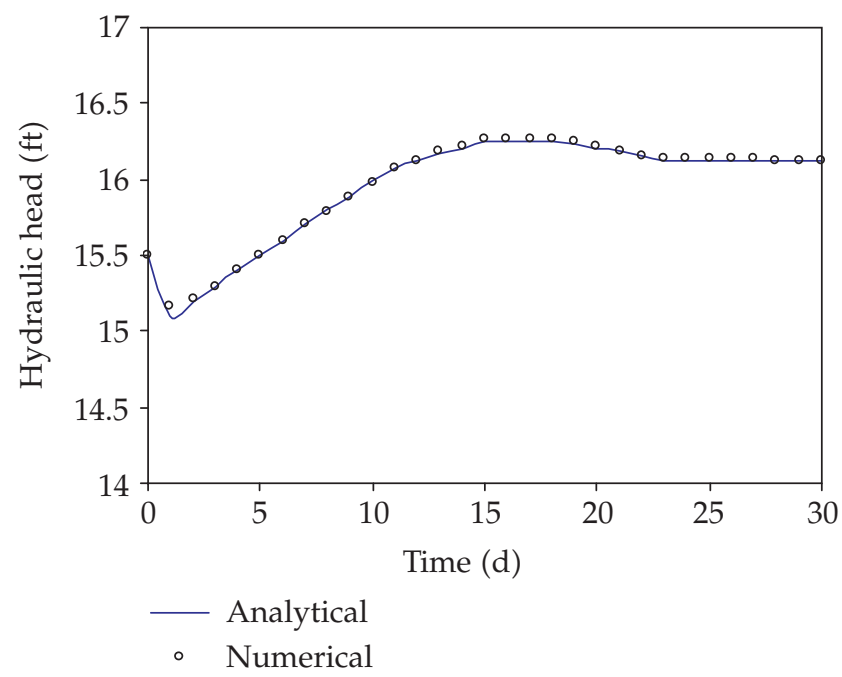

FIGURE 6.4. Finite element and analytical solutions for the mixed problem.

system. Therefore, the eigenvalues $\lambda_{i}, i=1,2,3, \ldots, p$, where $p=$ number of nodes, must be real and positive. This was verified numerically by computing the values for the coefficient matrix using Jacobi iteration [5]. The real positive values are investigated to determine the condition number (ratio of maximum-to-minimum eigenvalues) which gives a measure for the rate of convergence of the numerical solution for a symmetric positive definite system. The condition number is computed as $\aleph=\lambda_{\max } / \lambda_{\min } \approx 9$. The condition number indicates the rate of convergence of the conjugate gradient method because the required number of iteration varies with $\sqrt{\aleph}$.

\subsection{Free surface}

The free surface in the domain represents groundwater table in an unconfined condition. The solution of the variably saturated flow equation generates the time-varying free surface in the domain. In a porous medium, the free surface separates the unsaturated and the saturated zones. The free surface is located where the pressure head is zero. From the solution of the hydraulic heads, the pressure heads are obtained by subtracting the elevation head at the finite element grid points in the domain. The contour with zero-pressure head represents the free surface or the groundwater table in a porous medium. The locations of the free surface at different times for the Dirichlet and mixed problems are shown in Figures 6.6 and 6.7. The phreatic surface varies with time depending on the boundary conditions and recharge. In the mixed problem, a 
394 Variational analysis for simulating free-surface flows

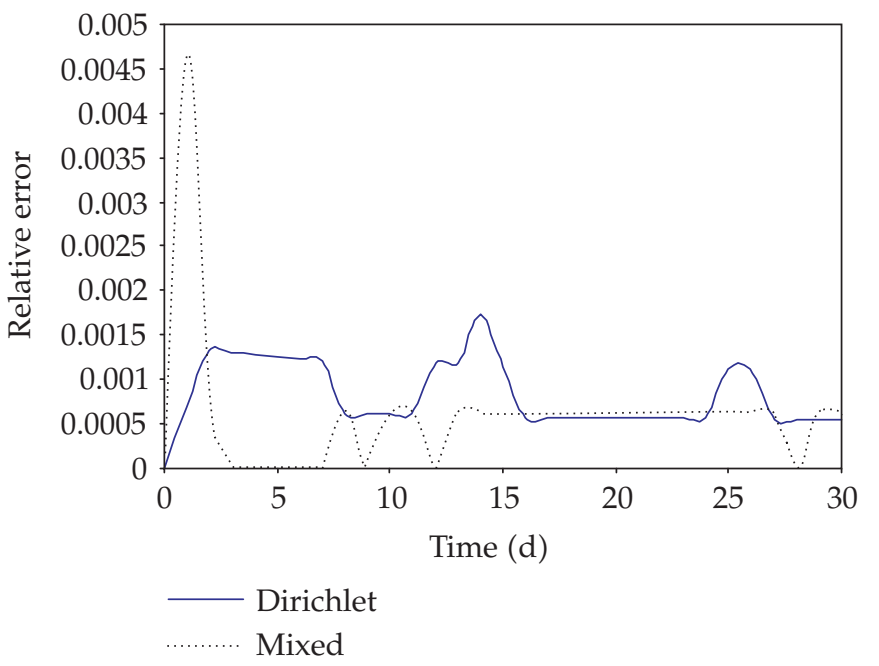

FIGURE 6.5. Time-varying relative errors for finite element solutions.

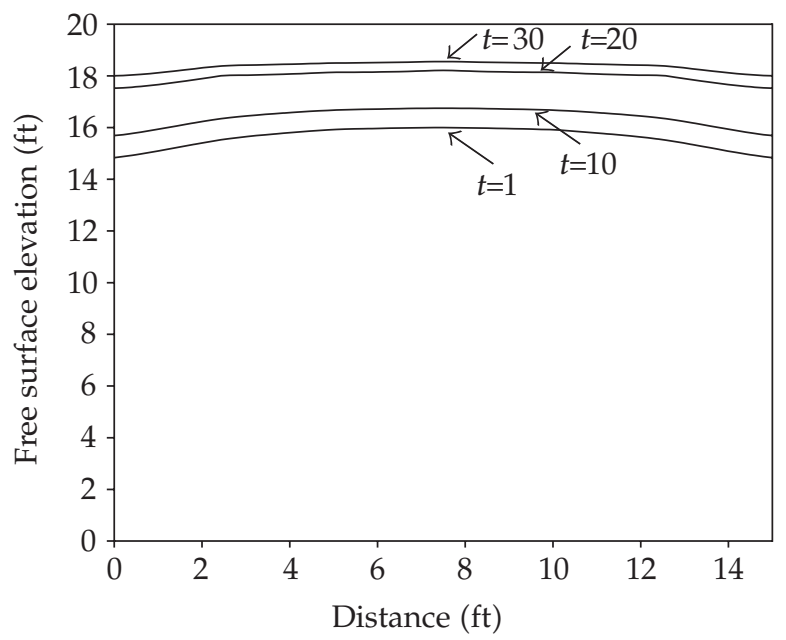

FigURE 6.6. Simulated free surface for the Dirichlet problem at different times.

time-varying Neumann flux $(\partial \varphi / \partial n=0.001)$ enters into the right boundary while the hydraulic head (Dirichlet boundary condition) varies with time on the left boundary. This is depicted by the simulated results in Figure 6.7. 


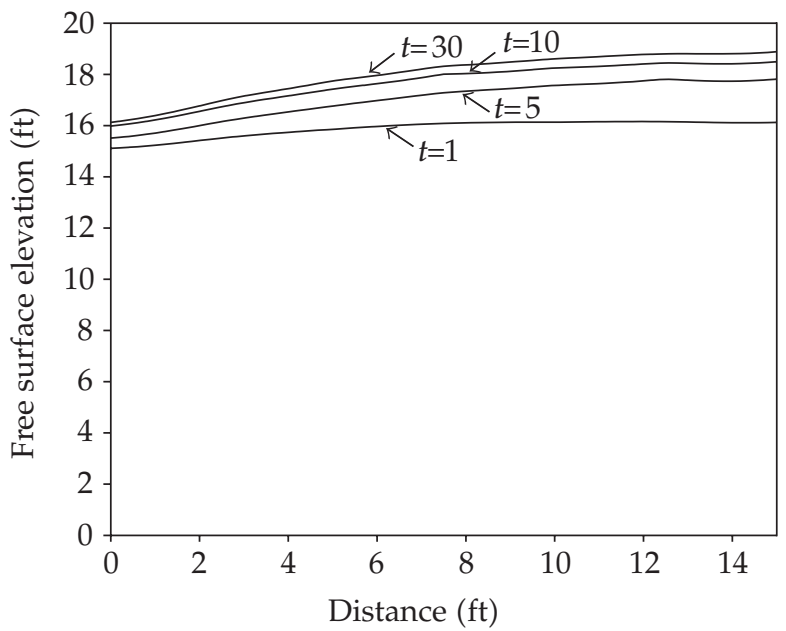

FIGURE 6.7. Simulated free surface for the mixed problem at different times.

\section{Summary and conclusion}

In this paper, variational formulations were developed for the finite element solutions of a parabolic equation describing variably saturated flow. The classical backward Euler method was used to obtain a fully discrete variational problem, which was transformed into a computer programme for generating time-varying hydraulic heads. The matrix characteristics and the growth of solutions are examined to establish the range of validity of the finite element formulation.

A symmetric positive definite system of equations is obtained and solved for the unknowns. The finite element solutions are compared with the analytical solutions. The finite element solutions agreed closely with the analytical solutions. The numerically simulated hydraulic heads at the grid points helped determine the free surface in the variably saturated flow domain. The successful application and implementation of the variational finite element analysis are found to be useful to simulate the variably saturated flow condition in the subsurface.

\section{References}

[1] S. Ahmed, Variational formulations for a parabolic partial differential equation describing variably saturated flow, Master's thesis, Department of Mathematics, The University of Tennessee, Tennessee, 2001.

[2] G. A. Baker, J. H. Bramble, and V. Thomée, Single step Galerkin approximations for parabolic problems, Math. Comp. 31 (1977), no. 140, 818-847.

[3] J. Bear, Hydraulics of Groundwater, McGraw-Hill, New York, 1979. 
396 Variational analysis for simulating free-surface flows

[4] K. Eriksson and C. Johnson, Error estimates and automatic time step control for nonlinear parabolic problems. I, SIAM J. Numer. Anal. 24 (1987), no. 1, 12 23.

[5] G. H. Golub and C. F. Van Loan, Matrix Computations, Johns Hopkins Studies in the Mathematical Sciences, Johns Hopkins University Press, Maryland, 1996.

[6] P. S. Huyakorn, P. F. Anderson, J. W. Mercer, and H. O. White Jr., Saltwater intrusion in aquifers: development and testing of a three-dimensional finite element model, Water. Resour. Res. 23 (1987), no. 2, 293-312.

[7] P. K. Jimack, A best approximation property of the moving finite element method, SIAM J. Numer. Anal. 33 (1996), no. 6, 2286-2302.

[8] C. Johnson, Numerical Solution of Partial Differential Equations by the Finite Element Method, Cambridge University Press, Cambridge, 1990.

[9] T. Kuppusamy, J. Sheng, J. C. Parker, and R. J. Lenhard, Finite element analysis of multiphase immiscible flow through soils, Water Resour. Res. 23 (1987), no. 4, 625-631.

[10] M. Luskin and R. Rannacher, On the smoothing property of the Galerkin method for parabolic equations, SIAM J. Numer. Anal. 19 (1981), no. 1, 93-113.

[11] S. P. Neumann, Saturated-unsaturated seepage by finite elements, ASCE J. Hydraul. Div. 99 (1973), no. 12, 2233-2250.

[12] A. Pandit and J. M. Abi-Aoun, Numerical modeling of axisymmetric flow, Journal of Ground Water 32 (1994), no. 3, 458-464.

[13] R. Srivastava and T.-C. J. Yeh, A three-dimensional numerical model for water flow and transport of chemically reactive solute through porous media under variably saturated conditions, Adv. in Water Res. 15 (1992), 275-287.

[14] V. Thomée, Negative norm estimates and superconvergence in Galerkin methods for parabolic problems, Math. Comp. 34 (1980), no. 149, 93-113.

[15] M. T. van Genuchten, A closed-form equation for predicting the hydraulic conductivity of unsaturated soils, Soil Sci. Soc. Am. J. 44 (1980), 892-898.

[16] T.-C. J. Yeh, R. Srivastava, A. Guzman, and T. Harter, A numerical model for water flow and chemical transport in variably saturated porous media, Journal of Ground Water 31 (1993), no. 4, 634-644.

Shabbir Ahmed: Big Cypress Basin, South Florida Water Management District, 6089 Janes Lane, Naples, FL 34109, USA

E-mail address: sahmed@sfwmd.gov

Charles Collins: Department of Mathematics, University of Tennessee, 121 Ayres Hall, 1403 Circle Drive, Knoxville, TN 37996, USA 


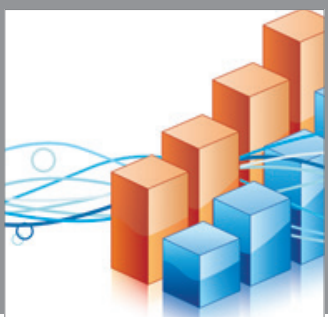

Advances in

Operations Research

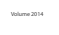

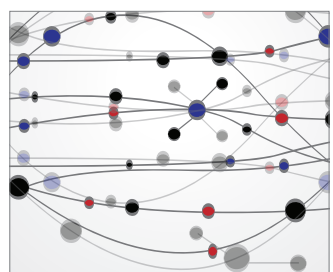

\section{The Scientific} World Journal
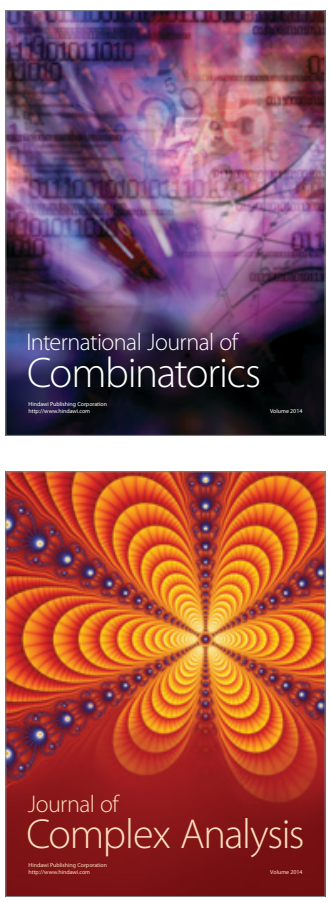

International Journal of

Mathematics and

Mathematical

Sciences
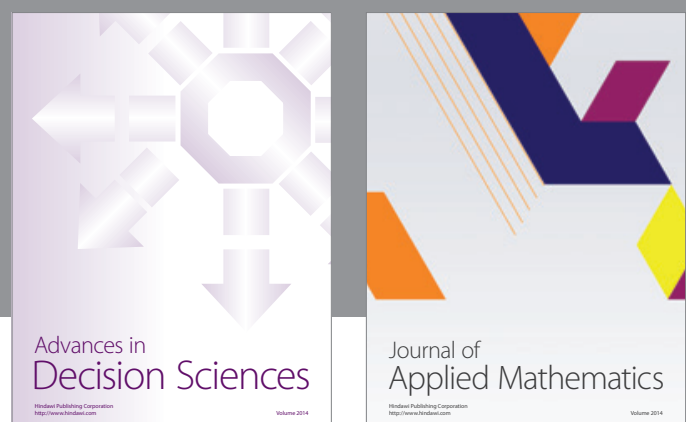

Journal of

Applied Mathematics
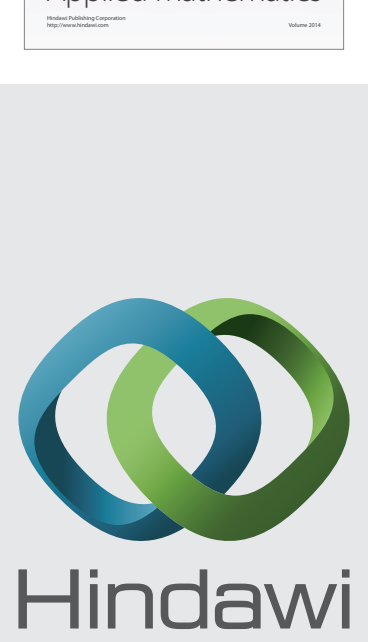

Submit your manuscripts at http://www.hindawi.com
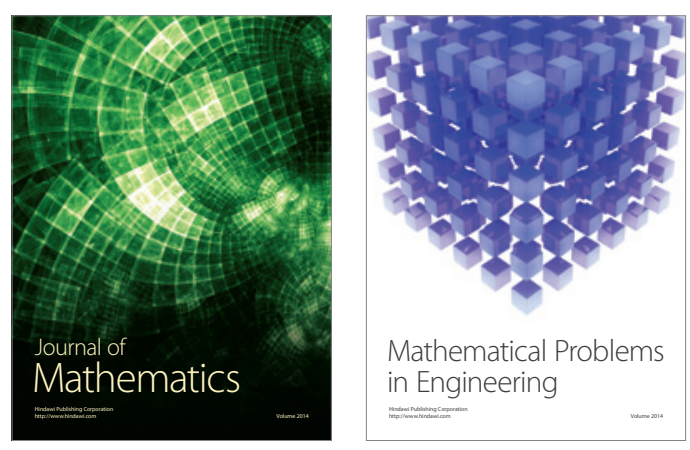

Mathematical Problems in Engineering
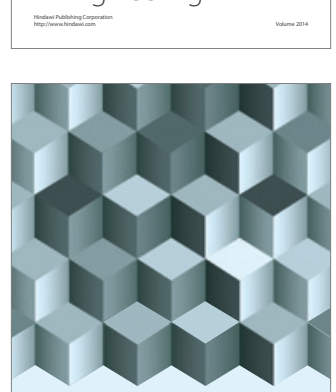

Journal of

Function Spaces
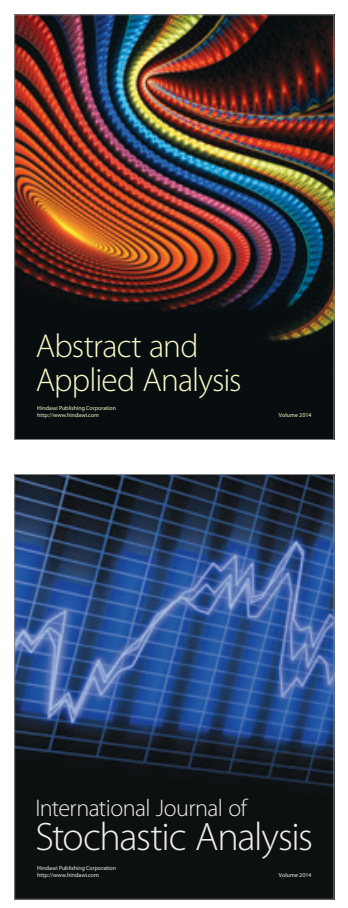

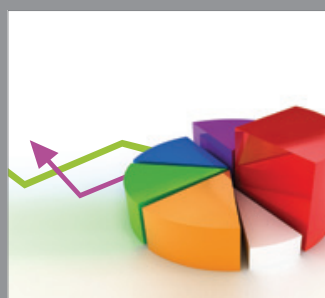

ournal of

Probability and Statistics

Promensencen
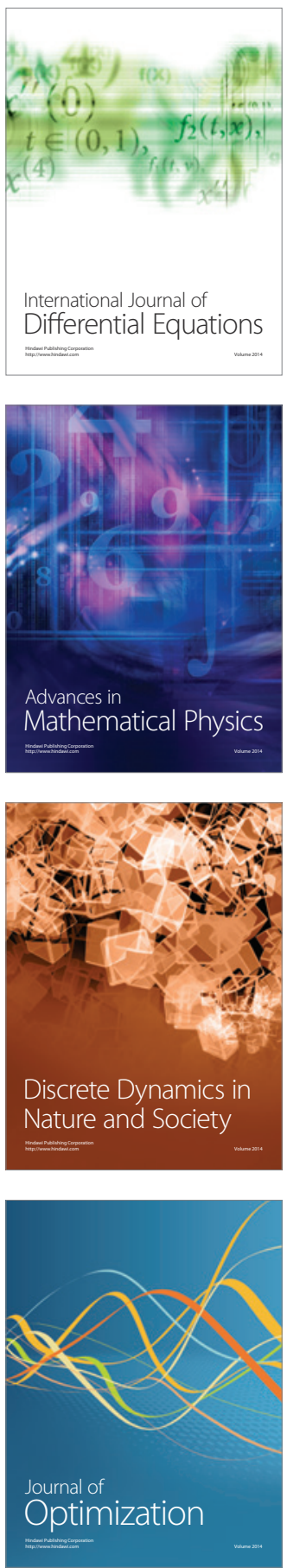